\title{
The Ideological Limitations on Translations of The Analects of Confucius: Inheritance and Transcendence
}

\author{
Yuezhu Fu \\ Peking University, Beijing, China \\ Email: 1693203821@qq.com
}

How to cite this paper: Fu, Y.Z. (2021) The Ideological Limitations on Translations of The Analects of Confucius. Inheritance and Transcendence. Open Access Library Journal, 8: e7908.

https://doi.org/10.4236/oalib.1107908

Received: August 31, 2021

Accepted: September 26, 2021

Published: September 29, 2021

Copyright $\odot 2021$ by author(s) and Open Access Library Inc.

This work is licensed under the Creative Commons Attribution International License (CC BY 4.0).

http://creativecommons.org/licenses/by/4.0/

\section{(c) (i) Open Access}

\begin{abstract}
There are many translation versions of The Analects of Confucius, including the first version by the foreign emissaries to China, and the following versions at home and overseas, as well as modern and contemporary works by Chinese translators. The translation of The Analects became more and more diversified, and the research on The Analects was more and more in-depth. However, the translation of The Analects of Confucius began with ideological constraints that is hard to get rid of. This paper will refer to three translation versions from James Legge, Waley and Ku Hung-ming, in order to analyze the Christian, orientalism and Confucian culture ideological limitations on the translation of The Analects of Confucius. The paper argues that the translation of The Analects must be based on inheriting classic translation principles, accurately understand the original texts, aptly add to the annotations, break the ideological limitation, so as to translate and spread essence of Confucian classics better.
\end{abstract}

\section{Subject Areas}

Linguistics

\section{Keywords}

The Analects of Confucius, Ideological, Principles of Translations, Inheritance and Transcendence

\section{1.《论语》翻译中意识形态上的限制}

\section{1. 里雅阁译本：基督教意识形态限制}

西方新教传教士马士曼(Joshua Marshman, 1768 1837)首次把《论语》直 
接从汉语翻译成英语, 题目译为 The Works of Confucius, 包括《论语》1 10 章, 译文正文共 725 页, 包括汉语原文与译文。汉语旁边还附有英语译音, 全书的最后一页是专有名词的拉丁文译名与英语 “译音” 的对照表, 1809 年 出版于塞兰坡 $[1]$ 。里雅阁是西方的传教士, 他从 1841 年开始翻译《论语》, 直到 1861 年完成翻译并出版。从《论语》英译之初, 就由西方传教士这一群 体肩负起了介绍东方文化的使命。西方传教士来华重要的目的是传播基督教, 他们翻译中国的经典文学, 一方面是为了了解中国的文化, 另一方面是为了 寻找中国文化与基督教的共同之处，便于更好地借助中国本土文学传播基督 教教义。所以在翻译中国典籍时也不可避免地赋予了《论语》基督教的意识 形态。

1)《论语・学而》第一篇:

子曰: “学而时习之, 不亦说乎? 有朋自远方来, 不亦乐乎? 人不知而 不愠, 不亦君子乎?”

(白话文解释: 学习后经常温习所学的知识, 不也很令人愉悦吗? 有志同 道合的人从远方来, 不也很高兴吗? 别人不了解我但我不生气, 不也是道德 上有修养的人吗? )

里雅阁将其译为:

The Master said, "Is it not pleasant to learn with a constant perseverance and application?

Is it not delightful to have friends coming from distant quarters?

Is he not a man of complete virtue, who feels no discomposure though men may take no note of him?"

其中，“学而时习之” 的 “习”，里雅阁将其理解为了 “学习加练习”, 将其翻译为 “perseverance and application” ，其中 perseverance 来源于动词 persevere。根据韦氏词典的解释, persevere 来自于中世纪英语, 表示 “to persist in a state, enterprise, or undertaking in spite of counterinfluences, opposition, or discouragement” , 并且还具有 “持续地蒙受神恩并最终达至荣耀的意义”。 这里强调通过不断地练习达到救赎。里雅阁在翻译 “习” 字时, 同时加上了 “application”, 符合 “践行、练习” 这一含义。很明显, 里雅阁是在基督教 层面诠释 “学习” 的含义, 主要强调儒家刻苦修身的过程, 来完成个人精神 的修炼, 来达到宗教意义上的救赎。里雅阁做为一个传教士, 其英译《论语》 的过程也是自身感悟宗教、获得顿悟的过程，所以在译文中会不断使用含有 宗教救赎色彩的词语。

2)《论语・为政》第四篇:

子曰: “吾十有五而志于学, 三十而立, 四十而不惑, 五十而知天命, 六十而耳顺，七十而从心所欲，不踰矩。”

(白话文解释: 我十五岁时, 便立定志向于学习之上; 三十岁时, 能立身 处世; 四十岁时, 可以免于迷惑; 五十岁时, 已经能够领悟天命; 六十岁时, 就可以顺从天命; 七十岁时, 终于能做到随心所欲而行, 且所为都能合于规 矩的境界了。)

里雅阁将其译为: 
The Master said, "At fifteen, I had my mind bent on learning.

At thirty, I stood firm.

At forty, I had no doubts.

At fifty, I knew the decrees of Heaven.

At sixty, my ear was an obedient organ for the reception of truth.

At seventy, I could follow what my heart desired, without transgressing what was right."

其中 “天命”, 里雅阁将其译为 “decrees of Heaven”, 回译过来就是 “天 堂的信条”。在基督教教义中, “heaven” 天堂据称为上帝在天的居所, 即上 帝之国, 也称天国 (Kingdom of God)。古今对于孔子 “知天命” 的理解, 主要 有两条路径: 一是把天命理解为个人所不能企及的意志力量, 或者说万事万 物之自然之理, 事物发展的内在规律。二是把天命理解为天所授予的使命, 知天命是指孔子自觉到了天所赋予的历史责任。这种解释主要是明清时代所 赋予的[2]。在里雅阁的译文中, 显然更倾向于第一种理解, 但是他把这种 “个 人所不能企及的意志力量” 直接译为了 “上帝的力量、来自天堂的信条” , 强调人一生的学习就是为了追求 “上帝的信条”。可见翻译中体现了浓厚的 基督教意识形态。

3)《论语・为政》第十四篇:

子曰: “君子周而不比, 小人比而不周。”

(白话文解释: 德行高尚的人以正道广泛交友但不互相勾结, 品格卑下的 人互相勾结却不顾道义。)

里雅阁将其译为:

The Master said, "The superior man is catholic and not partisan.

The mean man is partisan and not catholic."

里雅阁将 “比” 与 “周” 两个词分别译为 “catholic” 与 “partisan”, 这 是两个非常具有宗教色彩的词汇。“catholic” 作名词可以表示 “a member of the Roman Catholic Church” 即罗马天主教徒, 这个词作形容词时在牛津字典 上的解释为 “connected with all Christians or the whole Christian Church”。 “partisan” 表示 “prejudiced in favour of a particular cause” , 有 “盲目拥护” 的含义。实际上, 里雅阁是把君子和小人品德的比较与他们对于宗教信仰的 态度相等同了。所谓君子, 就是指忠于罗马天主教的人, 所谓小人就是盲目 拥护其他教派的人。

4)《论语・里仁篇第四》第十一则:

子曰: “君子怀德, 小人怀土; 君子怀刑, 小人怀惠。”

(白话文解释: 君子心怀的是仁德; 小人则怀恋乡土。君子关心的是刑罚 和法度, 小人则关心私利。)

The Master said, "The superior man thinks of virtue; the small man thinks of comfort. The superior man thinks of the sanctions of law; the small man thinks of favors which he may receive."

这一段中里雅阁将 “德” 译为 “virtue”, 将 “刑” 译为 “sanctions of law”, 其中 “virtue”一直是《圣经》中强调的 “美德、言行”, “sanctions” 又会 
让人想到 “末日审判”。里雅阁对这段话的翻译在潜移默化之中融入了基 督教的教义和故事, 把 “君子” 和 “小人” 思考的内容上升到宗教层面的 忓悔。

从上面的例子可以看出, 里雅阁在当时的《论语》英译本中加入了很多 自己对基督教的理解, 包括把儒家的哲学观念理解为基督教的教义, 把儒家 核心概念词与基督教中的教义相关联, 这体现了里雅阁在其译本中无法摆脱 基督教意识形态限制。

\section{2. 韦利译本: 东方主义情愫意识形态限制}

文学理论家、批评家萨义德在其著作《东方主义》中曾提到, 东方主义 有三重意义: “是一门学术研究的学科, 是一种思维方式, 是一种权利话语 方式。” [3] 其中, 东方是一个与 “西方” 相对的概念, 在西方文化概念中被 称为 “他者” , 东方愚昧、落后、封建而神秘, 同时也落后于西方。东方主 义的实质就是, 西方人研究中国文化时, 始终保持着一种高姿态, 潜意识中 以一种殖民者的心态, 把东方当成殖民地来看待, 是一种西方权力话语。东 方主义是西方权利话语, 依靠欧洲中心的观念对东方进行著作、翻译及编造, 进而丑化、弱化东方形象, 让在西方出生、成长的人, 无论多有想象力, 思 想多独特, 都不能摆脱这个话语的控制[4]。

英国汉学家亚瑟 - 韦利凭借着对中国文化的热爱, 翻译了《论语》。然 而在当时的西方殖民历史语境中, 韦利的翻译不可避免地带有东方主义情愫。 这主要体现在儒家思想核心概念词的翻译中。

1)《论语・里仁》中, 子曰: “朝闻道, 夕死可矣。”

(白话文解释: 早晨能够得知真理, 即使当晚死去, 也没有遗憾。)

韦利将其译为:

The master said, in the morning, hear the way;

in the evening, die content.

其中 “道” 是儒家思想非常核心的一个概念, 在这一句以及整篇论语的 翻译中, 韦利一直用一个词 “way” 来解释 “道”, 实际上在不同的语境之中, “道” 有不同的含义, 它也可以解释为 “good、 love、 humanity、 benevolence、 humaneness、goodness” 等。其中, “道千乘之国, 敬事而信, 节用而爱人, 使民以时”, 这里的 “道” 是指 “治理”, 用作了动词。韦利简单地将 “道” 译为 “way” 或者是 “ways”, 并没有传达出 “道” 的内涵, 显得单调而贫瘦, 这也容易让西方读者产生中国文化无趣、单调的误解。

2) 《论语・为政》中, 子曰: “君子周而不比, 小人比而不周。” 韦利将其译为:

The master said, a gentleman can see a question from all sides without bias. The small man is biased and can see a question only from one side.

3)《论语・述而》中, 子曰: “君子坦荡荡, 小人常戚戚。”

(白话文解释: 君子光明否落、心胸坦荡, 小人则斤斤计较、患得患失。) 韦利将其译为:

A true gentleman is calm and at ease, the small man is fretful and ill at ease. 
两句当中都出现了 “君子”、“小人”, 而韦利都将其译为 “gentleman”、 “small man”。但是结合语境, “君子周而不比, 小人比而不周” 出现在《论 语》的为政篇, 篇内的大多数话语都是在为政这个大的背景之下进行的扩展 和补充, 此处的 “君子” “小人” 更多含有政治意义, 而不是简单的 “gentleman” 或者 “small man”。第二处的 “君子坦荡荡, 小人长戚戚”, 更加强调的是两种品格的人的对比, 所以将 “君子” 翻译成 “the man of virtue” ，“小人” 翻译成 “the mean man” 会更加符合语境。反观韦利的翻 译用一个词汇概括所有的含义, 语义上十分模糊, 而且 “gentleman” 属于西 方语义中通俗易懂的词汇, 翻译过程中失去了中国文化中 “君子” 的内涵, 一味归化、简化翻译儒家核心概念词, 这表现出了韦利译本中的东方主义情 愫。另外在句法上韦利的译本句子结构非常简单, 缺乏原文句子结构的堆成 没, 而且 “a true gentleman is calm and at ease, the small man is fretful and ill at ease.” 中间用逗号链接, 不太符合英文的语法规范, 此处既然具有对比的含 义, 那么用连接词 “while” 会更加合适。

由此可见, 韦利对于很多富有儒家核心思想的文化负载词采取了 “省译” 或者 “复译” 的翻译策略, 选取简单直白的词汇难以表达儒家核心思想真正 的内涵。介于当时中国处于半殖民地半封建社会, 中国的文学地位也处于世 界文学的边缘, 很多经典作品在西方译者和读者看来价值不大, 因而在翻译 中存在淡化、弱化的东方哲学的色彩, 这体现了《韦利》译本中受到东方主 义意识形态限制。

\section{3. 幸鸿铭译本：儒家文化意识形态限制}

辜鸿铭是第一位英译《论语》的华人, 他在清末民国初英译《论语》, 打破了来华外国传教士和汉学家垄断中国经典翻译的局面。幸鸿铭(1898)在译 序中说: “里雅阁博士出版的《中国经典》译本迄今已有 40 余年。现在哪怕 对中国语言一无所知的任何人, 只要耐心阅读里雅阁博士的译文, 都会禁不 止感到其译文的确令人不满意。” 幸鸿铭翻译《论语》, 也是在阅读了里雅 阁等人的译文后进行的翻译, 他将自己认为不正确的地方进行了修改, 同时 特别强调突出了儒家传统思想的哲学内涵。但是由于自身经历和所信仰的观 念的限制, 㙜鸿铭的译本也未能完全打破意识形态的限制, 译本仍有不足。

幸鸿铭一生可以概括为 “四洋”, 他 “生在南洋、学在西洋、婚在东洋、 仕在北洋”。他学识渊博, 精通中国传统文化的同时, 又深入学习了西方哲 学、宗教。他虽然 “学在西洋”, 但却十分推崇和向往中国传统文化, 尤其 是儒家思想。幸鸿铭本人亦是一个封建文化的捍卫者, 身上处处体现着士大 夫的审美倾向, 他甚至在《中国人的精神》一书中大力提倡封建传统文化, 提倡妇女裹小脚, 提倡一夫多妻制度, 美化儒家文明, 力求将中国文明与西 方对等, 赢得西方的尊重。

首先, 辜鸿铭的译文有很多学习借鉴之处, 比如他为了传达原文的文化 精髓, 力求 “有机对等” , 在译文篇章上努力做到与原文对应, 在核心概念 的翻译中寻找西方文化相对等的概念进行替换。比如在《论语 - 为政》中, 子曰: “吾十有五而志于学, 三十而立, 四十而不惑, 五十而知天命, 六十 
而耳顺, 七十而从心所欲, 不踰矩。” 辜鸿铭将其译为: Confucius remarked, “At fifteen I have made up my mind to give myself up to serious studies. At forty I have formed my opinions and judgement. At forty I have no more doubts. At fifty I understood the truth in religion. At sixty I could understand what I heard without exertion. At seventy I could follow whatever my heart desired without transgressing the law." 这一句在句子结构的排列上与原文形式几乎对应, 而 且每一句开头都遵循原文 “三十” “四十” “五十” “六十” “七十”, 英 文形成了排比, 读起来非常具有节奏感。另外, 此句中, 喜鸿铭将 “知天命” 译为 “understood the truth in religion” ，把儒家思想中的 “天” 等同于西方 思想中的 “宗教” , 以此来弘扬传播儒家思想, 提高国外读者的接受度。但 是这里的天命更像是 “自然的规律” , 能不能将 “天命” 直接与 “religion” 等同, 还是值得商榷。

然而在整篇《论语》的翻译中, 辜鸿铭的译本也存在儒家文化意识形态 的限制。比如《论语 - 为政》第五则为:

孟懿子问孝。子曰: “无违。”

禁迟御, 子告之曰: “孟孙问孝于我, 我对曰, 无违。” 㚞迟曰: “何 谓也? ” 子曰: “生, 事之以礼; 死, 葬之以礼, 祭之以礼。”

(白话文解释: 孟懿子问孔子什么是孝道。孔子告诉他说: “不违背。” 柇迟为孔子驾车, 孔子告诉他说: “孟孙向我问什么是孝道, 我对他说, “不 违背, ”樊迟说: “什么意思呢? ” 孔子说: “活着, 以礼事奉, 死了, 以 礼安葬, 以礼祭祀。”)

喜鸿铭将其译为:

A noble of the Court in Confucius' native State asked him what constituted the duty of a good son. Confucius answered, "Do not fail in what is required of you."

Afterwards, as a disciple was driving him in his carriage, Confucius told the disciple, saying, "My Lord M-asked me what constituted the duty of a good son, and I answered, Do not fail in what is required of you'"

"What did you mean by that?" asked the disciple.

"I meant," replied Confucius, "When his parents are living, a good son should do his duties to them according to the usage prescribed by propriety; when they are dead, he should bury them and honour their memory according to the rites prescribed by propriety.”

从喜鸿铭的译文可以看出, 首先, 他没有直译人物的名字, 而是用身份 词语加以代替, 比如 “孟懿子”、“樊迟御”、“孟孙”, 以 “a noble”、

“a disciple”、“my lord” 来代替。实际上, 在整篇《论语》的译文中, 市 鸿铭在翻译中省略了很多人名的翻译, 这主要体现在孔子弟子的名字翻译上, 喜鸿铭几乎都用 “one disciple” “somebody” 这些词语来概括, 没有对孔子 的弟子进行介绍和身份阐释, 这其实也是享鸿铭意识中 “尊师重道” “尊卑 有序” 的儒家意识形态的影响。其次, 这一段的译文主要是在阐释儒家关于 
“孝” 的观点, “孝” 被喜鸿铭译为 “the duty of a good son.” 并且整篇是以 “he” 来代替 “孝” 的施动者。喜鸿铭默认为了 “孝” 是对于男性的要求, 受到三纲五常封建观念的影响, 把 “孝” 归为了 “儿子的义务”, 这就将女 性群体排除在外, 似乎女性是不需要对自己的父母 “尽孝”, 因为封建时代 女性归属于男性, “生要从父, 结婚要从夫, 夫死要从子”, 女性在政治经 济文化著作中没有发言权, 男性代表了女性, 因而 “孝” 只针对于男性。或 者说, 《论语》是一本封建时代写给男性修身治国齐家的书, 喜鸿铭默认地 将女性群体排除在了儒家哲学范畴之外。尽管幸鸿铭所处的时代已经发生了 “女权运动” 的思想变革, 但是辜鸿铭本人过于迷恋传统儒家文化, 不能理 智地区分精华和糟粕, 这也造成了他的《论语》英译本不可避免地带有儒家 意识形态的限制。

\section{2. 三个译本存在意识形态问题的原因}

综上所述, 论语的三个著名译本都或多或少体现了意识形态的限制。因 而在翻译中也存在很多不足。里雅阁的《论语》译本, 由于其基督教传教士 身份的限制以及介绍中国古典哲学的目的, 体现出浓厚的基督教意识形态色 彩。韦利自身是一名汉学家, 其《论语》译本简化了很多儒家传统哲学理念, 虽然易于西方读者理解, 但却在翻译中抹杀了《论语》的精髓。这也归因于 当时中西政治地位的不对等, 西方人对于中国文明同样抱有殖民者的态度, 体现了东方主义意识形态。幸鸿铭虽然是英译《论语》第一位华人, 学贯中 西, 志在以中国人的身份介绍儒家经典, 力求获得西方人的尊重, 然而他自 身盲目崇拜儒家文化, 在翻译中他将很多儒家经典的核心观念与西方宗教哲 学对等, 并且翻译思想中残存了儒家尊卑等级、重男轻女的封建糟粕, 这也 使得很多翻译偏离原文, 无法适应时代发展的需求。

\section{3. 《论语》翻译要超越意识形态限制}

笔者认为, 翻译应当超越意识形态的限制, 以客观、准确的视角翻译儒 家经典。比如, 在儒家核心概念词的翻译中, 既不要过度归化, 直接与西方 的概念等同, 也不要完全异化, 使得外国读者一头雾水。举例来说, “君子” 一词的翻译，如果直接等同于西方的 “gentleman”，未免失去了很多文化内 涵, 而且不利于西方读者了解 “君子”一词背后的民族品格。如果直接把君 子翻译成 “wise man”, 那又忽略了君子的道德品行, 另外古代不仅男人可 以称呼为君子, 女性也可以有君子之称, 所以不能只译为 “man”, 采用较 为中性的翻译, 在不同的语境中分清楚要强调君子的哪一部分品格, 比如强 调道德可以用 “the virtue”, 强调智慧可以用 “the intelligent”, 强调声望可 以用 “the respectful” 等等。不管是近代具有基督教意识形态、东方主义、儒 家文化意识形态的译本, 还是建国之后的现代《论语》译本, 比如著名语言 学家杨伯峻所翻译的, 都在一定程度上体现了意识形态的限制, 而要想打破 这种限制也是极其困难的。因此我们需要紧跟时代发展的需要, 推出能够被 外国读者更加广泛认可的译本。 


\section{4.《论语》翻译要传承的策略}

\section{1. 准确理解原文}

对于《论语》的翻译, 首先是要对原文理解准确。由于《论语》是由古 汉语写成, 已有五千多年的历史, 其中含有很多的通假字与多义词, 因此在 理解上很容易产生偏差。其实早在佛经翻译时期, 道安就认识到了佛经翻译 的 “无失本三不易” , 其中的 “三不易” 就是译经时有三种不容易的情况: 一是 “圣必因时, 时俗有易” ; 二是 “愚智天隔, 圣人匜阶” ; 三是 “今离 天年, 而以近意量裁” [5]。因此后人由于历史环境的变迁对于经典作品也很 难做到准确无误的理解。在《论语》的现代译本中, 杨伯峻的《论语译注》 和钱穆的《论语新解》是最可靠、最准确的两个版本。

在《论语.学而》篇中, 首句 “学而时习之, 不亦说乎? ”, 其中对于 “时” 和 “习” 两字的理解不尽相同。关于 “时” 的理解, “一指年岁言: 古人六 岁始学识字, 七八岁教以日常简单礼节, 十岁教书写计算, 十三岁教歌诗舞 蹈, 此指年为时。二指季节言: 古人春夏学诗月弦歌, 秋冬学书理涉猎, 此 指季节为时。三指晨夕言: 温习、进修、游散、休息、依时为之” [6]。钱穆 认同第三种说法 “按时、依时”。杨伯峻[7]谈到 “时” 在周秦时候若做副词 用, 等于《孟子・梁惠王上》 “斧金以时入山林, ” 的 “以时” , 指 “在一 定的时候” 或者 “在适当的时候”。此外, 《孟子 - 告子章句上》中有 “孔 子曰: 操则存, 舍则亡; 出入无时, 莫知其乡”。所以, 此处的 “时” 应该 是理解为 “时常” 比较准确。里雅阁将 “时” 英译为 “constantly”, 表示 “经常”。辜鸿铭将 “时” 和 “习” 结合在一起翻译, 将整个句子英译为

“Confucius remarked, it is indeed a pleasure to acquire knowledge and, as you go on acquiring, to put into practice what you have required.” 现代译者刘殿爵 将其译为 “时常”, 即 “at due intervals”, 是比较切合原意的。另外, 此句 中的 “习”, 究竟是 “复习”, 还是 “练习”, 实际上后期学者已经证明是 “练习” 更为妥当。《论语》各个译本中的讹误不再一一列举。纵观多个译 本, 现代译者刘殿爵的译本应该是最为准确的译本, 但是其中也存在对 “君 子” 等核心概念词翻译的争议之处。由此可见, 论语翻译中存在很多理解上 的误读, 这也是典籍翻译工作的重要难处。

\section{2. 适当添加注疏}

正式因为《论语》存在很多理解和翻译上的难题, 因而适当添加注疏也 是很有必要的。《论语》全书共 20 篇 492 章, 每一章都是针对不同的话题, 有其特定的语境。不仅在对论语原文阐释时需要适当添加注疏, 在翻译中也 要适当添加注释。比如在喜鸿铭译文中, 对于 “礼” 的翻译, 喜鸿铭进行了 注释 “Dr. LEGGA says of the Chinese word Li, which we have here translated 'art', that is a word not easily rendered in another language. On the other hand, Mr. B.H. CHAMBERLAIN, in his book Things Japanese, remarks that the Japanese language [China and Japanese have the same written language] has no genuine native word for 'art'.” 这样使得幸鸿铭对 “礼” 的翻译有了依据, 同时 
便于后人查证。另外在美国汉学家森舸澜的译文中, 他对每一句论语出现的 语境都进行了解释, 比如《论语 - 学而》第一篇, 森舸澜加入了这样一段话

"One of the central themes of this book is that learning has more to do with actual behaviour than academic theory, and that virtuous public behaviour as an adult is rooted in such basic familial virtues as filial piety and respect for elders (ti: 弟) (lit. “being a good younger brother')”, 对于整篇的孔子语录进行了解 释铺垫, 也是翻译中可取的策略之一。

\section{3. 注重传达文化精髓}

《论语》作为中国古代经典的哲学作品, 经历了五千年的历史至今依然 具有时代意义。然而针对于传统文化我们应当采取 “取其精华、去其糟粕” 的态度, 在翻译中将一些不符合时代发展的消极元素抹去, 比如孔子所强调 的尊卑、等级、男女有别的观念进行弱化, 将传统文化中积极的一面展现给 外国读者。在翻译中, 我们可以保留论语中含有中华传统哲学观念, 传统美 德的元素, 体现孔子 “仁爱” 的儒家思想内涵。

\section{5. 总结}

综上所述, 《论语》译本中不可避免带有意识形态的限制, 这是由翻译 所在的历史时期、翻译的目的以及译者的身份地位所决定的。不管是早期基 督教传教士的译本, 还是后期中国人的译本, 以及新中国成立后现代译者的 译本, 都没有完全打破意识形态的限制, 在翻译中多多少少地存在着个人认 知解读的狭险之处。而在全球化趋势加深以及中国综合国力不断提升的今天, 推动儒家经典《论语》译作走向世界已经成为时代的需求, 因而应当在准确 理解经典的基础上, 采用添加注疏等的策略, 打破意识形态限制, 注重传达 文化精髓, 使得《论语》能够为中外读者更好的接受。

本文也存在一定的不足: 对三个译本研究的深度不够, 例子可以更充实 些, 更具有说服力。最后作者提出的策略不够全面, 思考还可以更加深入。

\section{Conflicts of Interest}

The author declares no conflicts of interest.

\section{References}

[1] 李伟荣, 梁慧娜, 吴素馨. 《论语》在西方的前世今生[J]. 燕山大学学报, 2015(16): 1-8.

[2] 李振宏. 《论语》“四十而不惑, 五十而知天命”章是伪作 [J]. 清华大学学报, 2020, 35(1): 17-34.

[3] 爱德华. W. 萨义德. 东方学[M]. 王宇根, 译. 北京: 三联书店, 1999.

[4] 周宁. 东方主义: 理论与论争[J]. 厦门大学学报, 2003(1): 15-21.

[5] 陈福康. 中国译学理论史稿 $[\mathrm{M}]$. 上海: 上海外语教育出版社, 1996.

[6] 钱穆. 论语新解[M]. 北京: 新知三联书店, 2005.

[7] 杨伯峻. 论语译注[M]. 北京: 中华书局, 1980. 


\section{Appendix (Abstract and Keywords in Chinese)}

\section{《论语》翻译中意识形态上的限制一一传承与超越}

摘要: 《论语》译本繁多, 从起初最先来华传教士的翻译, 到后来中外译者 合译, 以及中国译者的现当代译本。译本越来越丰富, 同时相关《论语》研 究也越来越深入。然而, 从《论语》的翻译开始, 意识形态的限制就如影随 形, 难以摆脱。本文以里雅阁、韦利和幸鸿铭三个译本为参考, 尝试分析《论 语》英译中的基督教意识形态限制、东方主义情愫意识形态限制以及儒家文 化意识形态限制, 并且提出《论语》翻译必须在传承经典翻译原则的基础上, 准确理解原文, 适当添加注疏, 打破意识形态限制, 更好翻译和传播儒学经 典的主旨和精华。

关键词: 《论语》, 意识形态, 翻译原则, 传承与超越 